\title{
Use of Dense Stereo Matching for Existing Building Documentation: Comparative Analysis of Tools
}

\section{SIGRADI2018 TECHNOPOLITICAS \\ xxii congresso da sociedade iberoamericana de gráfica digital 22th conference of the iberoamerican society of digital graphics 07|08|09|novembro|2018 iau usp | são carlos | sp br}

\author{
Alana Bomfim de Araujo \\ UFBA | Brazil | alana.araujo18@gmail.com \\ Natalie Johanna Groetelaars \\ UFBA | Brazil | natgroet@ufba.br \\ Arivaldo Leão de Amorim \\ UFBA | Brazil | alamorim@ufba.br
}

\begin{abstract}
This paper presents a comparative study of Dense Stereo Matching (DSM) tools to generate point cloud from digital photogrammetric restitution. The capability of four different state-ofthe-art software systems as Photoscan (Agisoft), 3DF Zephyr Free (3Dflow), Remake (Autodesk) and Recap 360 (Autodesk) is examined to document a historical object. The main aspects compared are: processing time, export file formats, file size, quality and density of point clouds obtained from tools standard parameters. From the literature review, the analysis and the experiments, it is possible to evaluate the potential of DSM technique for the existing building documentation.
\end{abstract}

Keywords: Dense Stereo Matching (DSM); Digital photogrammetry; DSM tools; Point cloud; Triangular irregular network (TIN).

\section{INTRODUÇÃO}

O patrimônio cultural, sendo ele material ou imaterial, engloba diversas questões que identificam determinados grupos, como nacionalidade, etnia, religiões e alguns aspectos individualizados. No meio do contexto de globalização em que a sociedade contemporânea está inserida, o patrimônio encontra-se em um turbilhão de modificações, onde estes grupos se veem em um paradoxo entre a tradição e a busca incessante pelos necessários avanços tecnológicos (Amorim, 2017).

Em meio a essa busca, o patrimônio cultural vem se perdendo em função da deficiência de valor atribuído às tradições, devido à falta do sentimento de pertencimento e ao déficit da educação patrimonial, em favor da cultura moderna. O patrimônio cultural perdido permeia itens imateriais, como ritos, festas, tradições, crenças e lendas, e materiais, como obras de arte, livros e arquitetura. Esta última é a mais afetada quando se trata de perdas em favor dos avanços espaciais, tendo em vista as especulações e o crescimento desordenado das cidades.

Além das perdas provocadas pelos homens, como demolições, intervenções e depredações, existem as naturais como a ação de intempéries e desastres ambientais que podem causar grandes danos à configuração física de um objeto arquitetônico. Frente a tantos fatores de risco à arquitetura, mostra-se necessária a criação de ferramentas para o perduro da memória cultural. A documentação arquitetônica desempenha papel imprescindível para este fim, auxiliada e auxiliando a criação de identidade cultural.

Além do valor afetivo e simbólico, o levantamento preciso do objeto arquitetônico é imprescindível para diversas intervenções e aplicações em uma edificação, tendo como função ser base para elaboração de projetos de intervenção, restauro, para análises históricas detalhadas sobre a evolução do objeto arquitetônico e avaliações das tecnologias construtivas empregadas (Oliveira, 2008).

No Brasil, grande parcela do patrimônio cultural ainda não está devidamente documentada; e para tal, seria necessário o desenvolvimento de métodos que auxiliem o processo, com maior rapidez e precisão.

A documentação arquitetônica esteve, durante muito tempo, associada ao levantamento cadastral por medição direta. No entanto, em função do grande desenvolvimento tecnológico, agregaram-se novas ferramentas que permitem auxiliar a obtenção de diversos tipos produtos. Dentre as novas tecnologias, é possível citar panoramas fotográficos, vídeos, fotogrametria digital, $3 D$ laser scanning, Dense Stereo Matching (DSM), que cada vez mais facilitam a obtenção de produtos como fotos retificadas, ortofotos, desenhos e modelos geométricos. Deste modo, estas tecnologias apresentam extrema importância para preservação do patrimônio cultural, para uso em museus virtuais e outros (Nikolov; Madsen, 2016). 
Este artigo discute a utilização do Dense Stereo Matching no levantamento cadastral de edificações existentes, e faz a análise comparativa de diferentes ferramentas para DSM. Apresenta-se aqui uma parte do material produzido na pesquisa de Iniciação Científica, com o plano de trabalho intitulado "Estudo comparativo de ferramentas para restituição fotogramétrica”, relacionado ao projeto de pesquisa "Desenvolvimento de Métodos para auxiliar as etapas de levantamento e geração de modelos BIM de edificações existentes", desenvolvido no Laboratório de estudos Avançados em Cidade, Arquitetura e tecnologias Digitais (LCAD) da Faculdade de Arquitetura da Universidade Federal da Bahia (FAUFBA). As ferramentas comparadas foram o Photoscan (programa comercial da Agisoft), o Remake e Recap 360 (ferramentas de processamento online da Autodesk), e o programa 3DF Zephyr Free da 3Dflow (a versão gratuita da empresa 3Dflow).

\section{RESTITUIÇÃO FOTOGRAMÉTRICA}

A Fotogrametria é uma técnica de levantamento cadastral realizada através de fotos, sendo aplicada na Arquitetura desde meados do século XIX, quando foi observado, pelo arquiteto alemão Meydenbauer, o seu potencial para a documentação arquitetônica (Groetelaars, 2004). Esta técnica foi, por muito tempo, pouco desenvolvida, devido à necessidade de grande especialização para o manuseio dos equipamentos necessários à época e de recursos para obtenção dos instrumentos, para manutenção e espaço para mantê-los, visto que eram grandes máquinas com rebuscados sistemas de lentes.

Entre as décadas de 1980 e 1990, com o crescente desenvolvimento dos computadores, a fotogrametria passa a ser mais difundida; não mais a analógica, e sim a fotogrametria com auxílio de computadores, a digital, como pode ser observado na Figura 1.

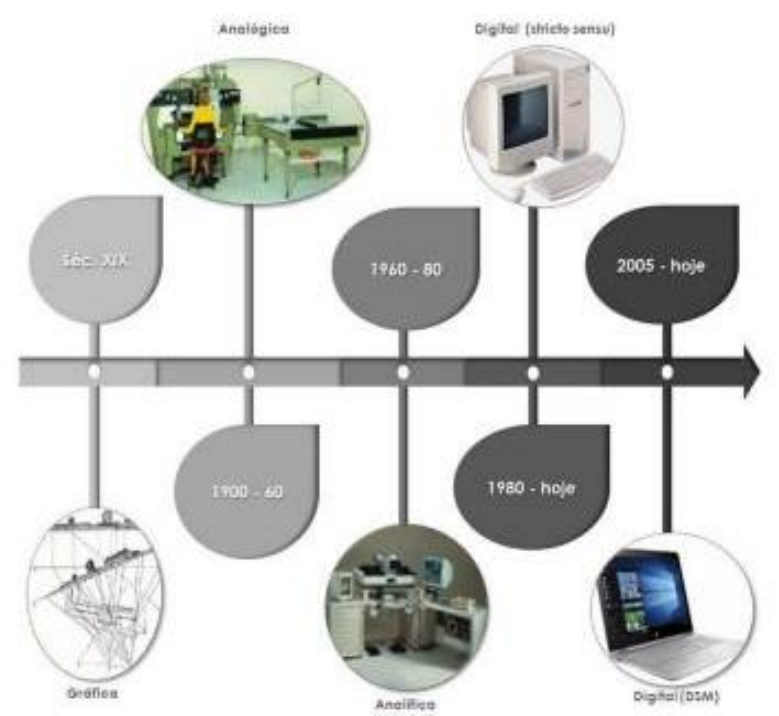

Figura 1: Linha do tempo da Fotogrametria. Fonte: autores.

Desde os anos 2000, a criação de softwares específicos para a fotogrametria ganhou atenção de desenvolvedores, e consequente popularização desse método de levantamento de edificações.
Por volta dos anos 2005-2010, surgiram ferramentas mais automatizadas, permitindo a obtenção de produtos com elevados níveis de precisão, de modo mais rápido que a Fotogrametria Digital stricto sensu.

A Fotogrametria stricto sensu está associada a métodos de restituição mais interativos, realizada a partir da identificação de pontos homólogos pelo usuário, sendo mais indicada para levantamentos de objetos poliédricos, com feições geométricas bem definidas, gerando como produtos primários, modelos geométricos de superfícies (fotorrealísticos ou não), desenhos e ortofotos.

Com os avanços tecnológicos ocorridos nas áreas da fotogrametria digital, da visão computacional e em equipamentos, foi possível o desenvolvimento de técnicas de obtenção de modelos de nuvem de pontos de grande qualidade a partir de fotografias (Remondino et al., 2014). O Dense Stereo Matching - DSM (também denominado de Structure from Motion) é um exemplo disto. O DSM representa o estado da arte em relação às técnicas automatizadas para restituição fotogramétrica, permitindo a obtenção de modelos de objetos com grande nível de complexidade, com pequena interação humana.

O DSM funciona a partir da associação de feições homólogas (conjunto de pixels homólogos) em diferentes fotos através do reconhecimento de padrões e do princípio da estereorrestituição. Para isso, são necessárias imagens com grande área de sobreposição e pequena angulação entre si, características fundamentais para a tomada fotográfica. Como produtos primários do DSM, tem-se o modelo geométrico tipo "nuvem de pontos" e/ou a malha triangular irregular (Triangular Irregular Network - TIN), que varia de acordo com a ferramenta digital utilizada para o processamento dos dados (Groetelaars, 2015).

Para que a tomada de fotos seja adequada para a obtenção de modelos a partir do DSM, ou seja, para que haja a correlação de pixels homólogos, é necessário um planejamento do levantamento e cumprimento de alguns requisitos, descritos a seguir:

- cada objeto deve ser fotografado em, no mínimo, três posições diferentes, com angulação menor que 15 graus entre elas;

- deve-se respeitar a relação $0,1<B / L<0,5$, onde $B$ é a distância entre as estações de tomada fotográfica e $L$ é a distância da câmera para o objeto. Para Hullo, Grussenmeyer e Fares (2009) essa relação deve estar entre 0,3 e 0,1, para garantir precisão geométrica e, ao mesmo tempo, não produzir grandes variações entre os conjuntos de pixels a serem comparados, o que dificultaria ou impediria a correlação automática dos mesmos;

- a textura do objeto não deve ser uniforme, deve haver variação de coloração ou apresentar alguma rugosidade, variação de relevo, etc.;

- todas as fotos devem ter boa iluminação e nitidez, devendo-se realizar o levantamento fotográfico sob as mesmas condições de luminosidade;

- a tomada fotográfica deve ser realizada em curto espaço de tempo, para evitar a variação de luminosidade e sombra em ambientes externos e 
internos, não sendo possível o uso de flash (localização variável da iluminação).

É necessário que o planejamento seja cuidadoso, respeitando esses aspectos para reduzir a probabilidade de encontrar problemas no modelo geométrico obtido, como nuvem de pontos esparsa, baixa precisão dos pontos, distorções, presença de ruídos e/ou oclusões.

\section{METODOLOGIA}

Com o intuito de investigar as melhores aplicações das ferramentas automatizadas para restituição fotogramétrica, iniciou-se $o$ processo de análise e comparação das etapas de processamento e dos produtos gerados. As ferramentas analisadas foram Autodesk Recap 360, Autodesk Remake, Agisoft Photoscan e 3DF Zephyr Free.

O objeto utilizado para a comparação das ferramentas foi a porta do claustro da Igreja e Convento Santo Antônio do Paraguaçu, às margens do Rio Paraguaçu, no povoado de mesmo nome em Cachoeira (BA). Os processamentos foram executados em computador com processador core i7 vpro e memória RAM de 16 Gb.

Foram utilizadas seis imagens obtidas por uma câmera Sony, modelo DSC-HX7V, com resolução de $4.608 \times$ 3456 pixels. A tomada fotográfica foi em formato ligeiramente circular (com pequena angulação entre as fotos), sendo realizada em ambiente iluminado por luz natural difusa, evitando-se o sombreamento de partes do objeto (Figura 2). As fotos possuíam boa nitidez e alta resolução, devido à aproximação com o objeto.
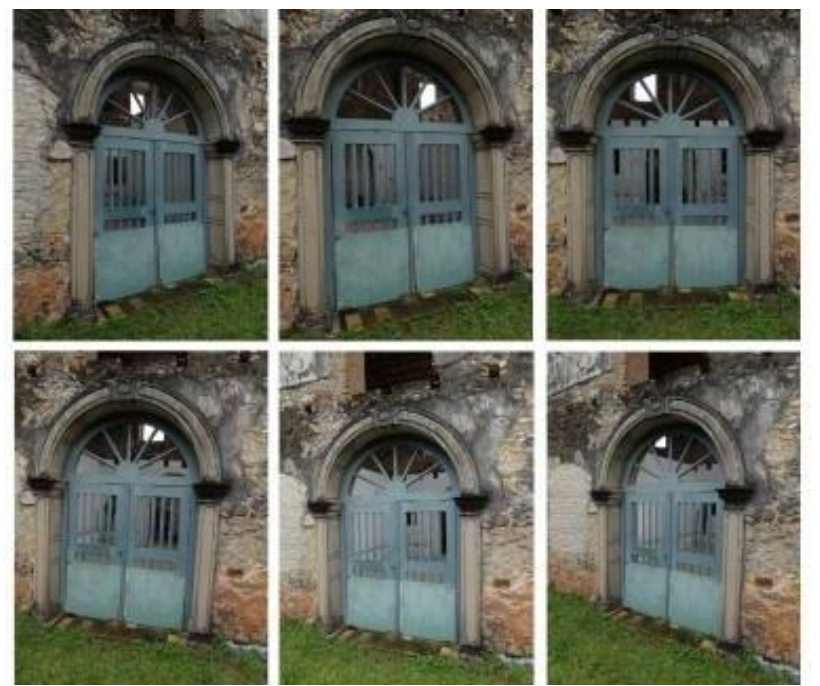

Figura 2: Diversos ângulos da porta do claustro da Igreja e Convento Santo Antônio do Paraguaçu. Fonte: autores.

\section{AUTODESK RECAP 360 E REMAKE}

O primeiro experimento foi realizado no Recap 360, uma ferramenta da Autodesk que realiza o processamento online. A velocidade de processamento não sofre grande influência das configurações da máquina utilizada, e sim da velocidade da internet e dos servidores da Autodesk. Há pouco controle do usuário com relação à definição dos parâmetros para criação de nuvens de pontos. Um dos parâmetros que devem ser escolhidos é a qualidade do modelo: standard e high quality. Nesse caso, usou-se o parâmetro standard (qualidade padrão), disponível para o modo gratuito.

O produto obtido no Recap 360 foi um modelo em malha triangular extremamente completo, com grande riqueza de detalhes e pouca distorção, como pode ser observado na Figura 3. O tempo necessário para a criação deste modelo foi 18 min e $2 \mathrm{~s}$, incluindo o tempo de upload e processamento das imagens para gerar a malha TIN.

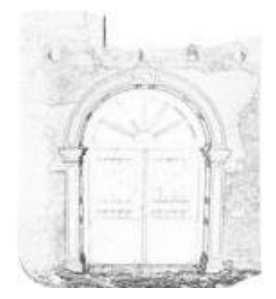

(a)

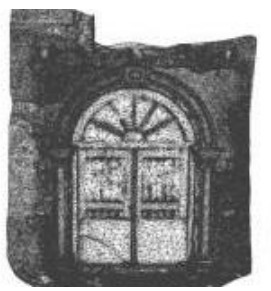

(b)

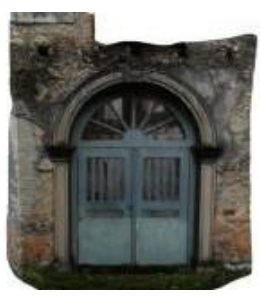

(c)
Figura 3: Produtos gerados no Autodesk Recap 360: (a) nuvem de pontos monocromática; (b) modelo em malha TIN; (c) modelo em malha TIN com textura aplicada. Fonte: autores.

O Recap 360 foi desenvolvido para permitir a edição e visualização de nuvens de pontos, seja pela varredura a laser, seja por DSM. Essa ferramenta foi criada também para permitir a geração de modelos geométricos em malhas TIN e nuvem de pontos de objetos, edifícios e até mesmo de grandes áreas urbanas a partir de fotografias, de modo gratuito, através do processamento das fotografias enviadas aos servidores da Autodesk.

Outra ferramenta da Autodesk também voltada à fotogrametria digital é o Autodesk Remake, que foi descontinuado em setembro de 2017. O Remake, apesar de similar ao Recap 360, foi desenvolvido com a função de gerar malhas TIN (ou nuvens de pontos) a partir de fotos e permitir a edição das mesmas, através de comandos para limpeza, edição e impressão destes modelos. O Remake foi uma evolução do Autodesk Memento.

Após atualização realizada ao final de 2017, as funções do Recap 360 e do Remake foram transferidas para o Recap Photo, ferramenta inclusa no Recap Pro, última versão da Autodesk, em que há cobrança pelo serviço de processamento online das fotos. A versão gratuita desse serviço é possível para uso educacional, onde há limitação do número do fotografias a serem processadas (mínimo de 20 e máximo de 99 fotos).

A criação do modelo no Remake foi mais rápida que no Recap 360, sendo necessários $9 \mathrm{~min}$ e $43 \mathrm{~s}$ para o processamento. A velocidade do Remake não afetou a qualidade do produto em relação ao programa anterior, uma vez que permitiu gerar um modelo em malha TIN com alta resolução e qualidade na texturização, como é possível ver na Figura 4.

Observa-se nos modelos gerados no Recap 360 e Remake, que há menor densidade da malha nas áreas mais planas e com texturas mais homogêneas e maior densidade nas áreas mais complexas, com maior rugosidade e diferentes texturas. 


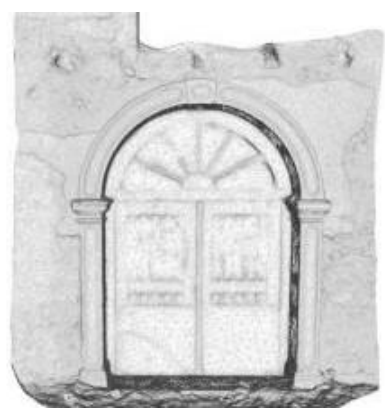

(a)

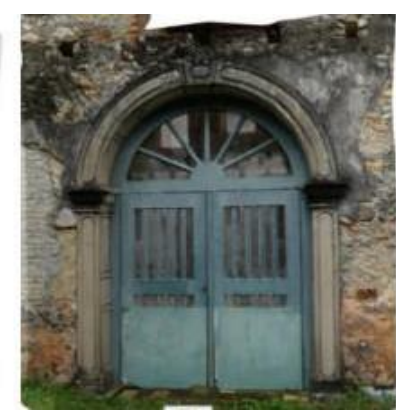

(b)
Figura 4: Produtos gerados no Autodesk Remake: (a) modelo em malha TIN; (b) modelo em malha TIN com textura aplicada. Fonte: autores.

Observa-se nos modelos gerados no Recap 360 e Remake, que há menor densidade da malha nas áreas mais planas e com texturas mais homogêneas e maior densidade nas áreas mais complexas, com maior rugosidade e diferentes texturas.

\section{AGISOFT PHOTOSCAN}

O experimento seguinte foi feito no Agisoft Photoscan. O Photoscan é um software para Dense Stereo Matching, que gera nuvens de pontos a partir de fotografias. É uma ferramenta proprietária, comercial, de interface simples, tendo sua versão Professional Edition sido disponibilizada gratuitamente por um período de 30 dias para testes.

É uma ferramenta que pode ser manipulada através de processos automatizados, facilitando a utilização para iniciantes no campo da fotogrametria, todavia oferece também diversas opções de ajuste e parâmetros, interessantes para o trabalho de especialistas produzindo diferentes tipos de dados.

Com as mesmas imagens, o Photoscan realizou o processamento e gerou modelos em nuvem de pontos, malha TIN e textura de elevada qualidade (Figura 5), em 3 min e $26 \mathrm{~s}$.

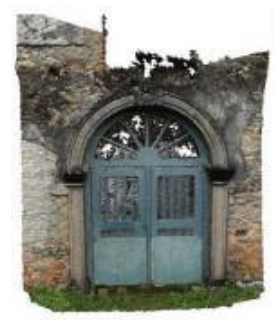

(a)

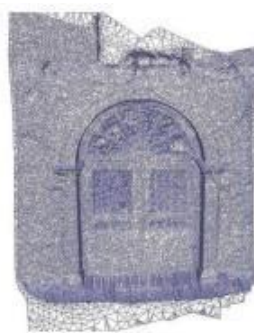

(b)

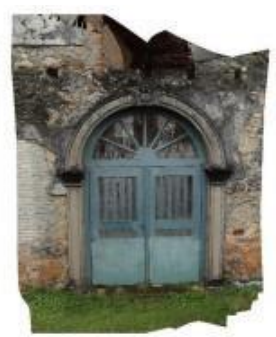

(c)
Figura 5: Produtos gerados no Agisoft Photoscan: (a) nuvem de pontos com a cor do ponto; (b) modelo em malha TIN; (c) modelo em malha TIN com textura aplicada. Fonte: autores.

\section{DF ZEPHYR FREE}

O processamento seguinte foi realizado no 3DF Zephyr Free da 3Dflow, uma ferramenta proprietária, de uso gratuito (na versão Free não há limitação de tempo de uso), com processamento automático, utilizada tanto para reconstrução de pequenos objetos quanto para a criação de modelos de terreno e de edificações.
Possui uma interface amigável e seu processamento automático não necessita de alvos codificados, realizando também restituições sem a calibração prévia das câmeras. Lançado em 2017, a versão gratuita do Zephyr apresenta algumas restrições em relação à versão profissional (comercial), como a limitação da quantidade de fotos a serem processadas (no máximo 50 imagens para cada modelo) e disponibilização de apenas ferramentas básicas para edição e exportação.

Além da versão gratuita, a 3DFlow oferece mais três versões comerciais, a versão Lite, com restrição de 500 imagens, Pro e Aerial, ambas sem restrições em relação à quantidade de imagens. As versões Pro e Aerial, as mais desenvolvidas da ferramenta, contam com suporte para Laser Scanning, ferramentas avançadas para edição, possibilidade de uso de pontos de controle e permite gerar produtos em diversos formatos de exportação.

Para o processamento das imagens, o Zephyr Free requer a inserção de parâmetros, como qualidade da malha, quantidade de pontos da nuvem de pontos e produtos desejados. As definições de qualidade foram mantidas no padrão fornecido pelo programa e os produtos escolhidos foram nuvem de pontos, malha TIN e malha TIN texturizada, como pode ser visto na Figura 6 , resultados de um processamento que durou $16 \mathrm{~min}$ e 18 s.

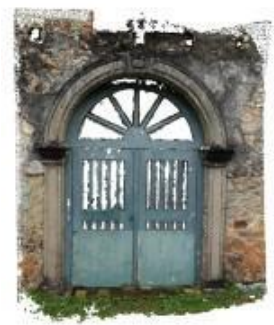

(a)

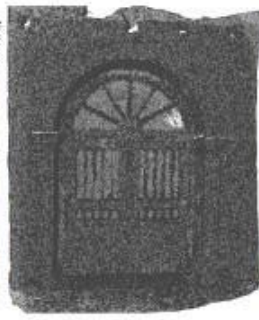

(b)

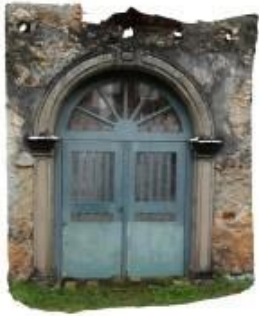

(c)
Figura 6: Produtos gerados no 3DF Zephyr Free: (a) nuvem de pontos com a cor do ponto; (b) modelo em malha TIN; (c) modelo em malha TIN com textura aplicada. Fonte: autores.

\section{RESULTADOS}

Para a análise comparativa das ferramentas, foram avaliados os seguintes aspectos:

- análise visual dos produtos gerados (nuvem de pontos e malha TIN);

- fluxo de trabalho;

- formatos de exportação;

- tempo de processamento;

- tamanho dos arquivos;

- quantidade de vértices e de faces triangulares dos modelos gerados.

Com relação à análise visual dos produtos gerados, percebe-se a elevada qualidade do modelos gerados nas quatro ferramentas utilizadas, tanto no que se refere à quantidade de informação registrada, à precisão e à qualidade da textura fotorrealística aplicada à malha TIN. 
Nos modelos gerados no PhotoScan e 3DF Zephyr Free verifica-se que há homogeneidade na densidade da malha (independentemente de serem áreas mais planas, complexas ou com mais texturas), o que não ocorre nos modelos do Remake e Recap 360, em que há diferenciação na densidade da mesma.

Em se tratando do fluxo de trabalho, há diferenças das etapas e do grau de interferência do usuário nas ferramentas, como pode ser visto na Figura 7. Em geral, as ferramentas online apresentam menor interação e menor controle de resultados, sem exigir grande capacidade de processamento do computador utilizado.

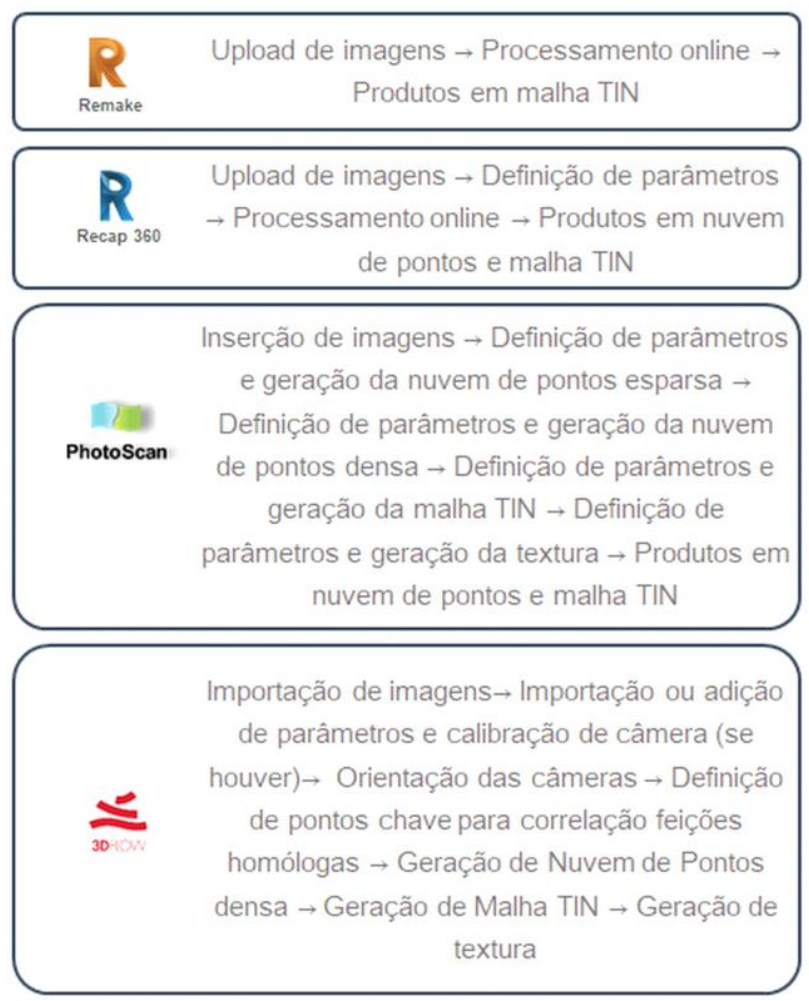

Figura 7: Etapas de processamento para obtenção de produtos das ferramentas Recap 360, Remake, Photoscan e Zephyr Free. Fonte: autores.

Salienta-se que em todos os programas analisados, os processamentos e parâmetros foram realizados na opção padrão (ou standard) já imputada.

Outros aspectos observados foram os formatos de exportação do produtos tanto para modelos do tipo nuvem de pontos ou malha TIN, como outros tipos de produtos, conforme ilustrado no Quadro 1.

Pelo Quadro 1, observa-se uma variedade muito maior de formatos de exportação da ferramenta Photoscan Profissional Edition. A versão 3DF Zephyr Free apresenta grandes limitações de formatos, não permitindo exportar modelos do tipo nuvem de pontos.

No entanto, é importante ressaltar que as versões mais completas do 3DF Zephyr (Pro e Aerial) apresentam uma variedade muito maior de formatos de exportação do que a versão gratuita. É possível exportar modelos de nuvem de pontos nos formatos PTS, PTX, XYZ, TXT, LAS e E57; e modelos em malha TIN nos formatos PLY, OBJ, FBX,
STL, U3D, Collada, além de permitir gerar arquivos no formato PDF3D.

Quadro 1: Formatos de exportação das ferramentas Recap 360, Remake, Photoscan e Zephyr Free.

\begin{tabular}{|c|c|c|c|c|}
\hline & Recap 360 & Remake & Photoscan & Zephyr Free \\
\hline 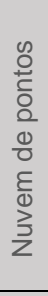 & $\begin{array}{l}\text { E57 } \\
\text { PCG } \\
\text { PTS } \\
\text { RCP } \\
\text { RCS }\end{array}$ & $\begin{array}{l}\text { E57 } \\
\text { PCG } \\
\text { RCP } \\
\text { PTS } \\
\text { RCS }\end{array}$ & $\begin{array}{l}\text { ASTM } \\
\text { CL3 } \\
\text { E57 } \\
\text { LAS } \\
\text { LAZ } \\
\text { OC3 } \\
\text { PTS } \\
\text { XYZ } \\
\end{array}$ & \\
\hline 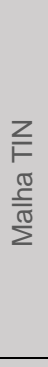 & OBJ & $\begin{array}{l}\text { FBX } \\
\text { OBJ } \\
\text { RCM } \\
\text { STL }\end{array}$ & $\begin{array}{l}\text { 3DS } \\
\text { VRML } \\
\text { COLLADA } \\
\text { DXF } \\
\text { FBX } \\
\text { KMZ } \\
\text { OBJ } \\
\text { PLY } \\
\text { STL } \\
\text { U3D } \\
\end{array}$ & $\begin{array}{l}\text { OBJ } \\
\text { PLY }\end{array}$ \\
\hline $\begin{array}{l}\text { \& } \\
\text { O로 } \\
\text { Oे }\end{array}$ & & & PDF & PDF \\
\hline
\end{tabular}

Com relação às outras questões analisadas, pode-se resumi-las no Quadro 2, que mostra os principais dados sobre os processamentos realizados nas ferramentas para DSM e algumas características dos produtos obtidos, como quantidade de vértices, faces e tamanhos dos arquivos gerados.

Quadro 2: Resultados do processamento nas ferramentas Recap 360, Remake, Photoscan e Zephyr Free da porta do claustro da Igreja e Convento Santo Antônio do Paraguaçu.

\begin{tabular}{c|cccc}
\hline \multicolumn{1}{c}{$\begin{array}{c}\text { Recap } \\
360\end{array}$} & Remake & Photoscan & $\begin{array}{c}\text { 3DF } \\
\text { Zephyr }\end{array}$ \\
\hline $\begin{array}{c}\text { Tempo total de } \\
\text { processa- } \\
\text { mento }\end{array}$ & $\begin{array}{c}18 \mathrm{~min} \\
02 \mathrm{~s}\end{array}$ & $\begin{array}{c}9 \mathrm{~min} \\
43 \mathrm{~s}\end{array}$ & $\begin{array}{c}3 \mathrm{~min} \\
26 \mathrm{~s}\end{array}$ & $\begin{array}{c}16 \mathrm{~min} \\
18 \mathrm{~s}\end{array}$ \\
$\begin{array}{c}\text { Quantidade de } \\
\text { vértices }\end{array}$ \\
$\begin{array}{c}\text { Quantidade de } \\
\text { faces }\end{array}$ \\
$\begin{array}{c}\text { triângulares } \\
\begin{array}{c}\text { Tamanho do } \\
\text { arquivo }\end{array}\end{array}$ & 247.931 & 247.931 & 84.223 & 269.817 \\
\hline
\end{tabular}

Verifica-se que existem diferenças consideráveis nos resultados de processamento a depender da ferramenta utilizada. O decurso do tempo, a quantidade de vértices e o tamanho dos arquivos na maioria dos dados coletados apresentam grandes diferenças que influenciam na escolha da ferramenta para determinados fins. 
Mesmo com menor consumo do processamento do computador utilizado, as ferramentas online, Remake e Recap 360 necessitaram de mais tempo para gerar os produtos, tendo a última apresentando o processamento mais lento dentre as ferramentas analisadas. O Photoscan mostrou-se superior nesse item, levando um pouco mais de um terço do tempo requerido pelo Remake.

O modelo gerado no 3DF Zephyr Free foi o que apresentou a quantidade um pouco maior de vértices $e$ triângulos em relação às outras ferramentas, mas acabou gerando um arquivo extremamente grande, de aproximadamente $307 \mathrm{Mb}$.

A ferramenta que permitiu gerar um modelo com menor tamanho de arquivo, mas mantendo a qualidade do produto foi o Autodesk Remake.

\section{DISCUSSÃO}

Comparando as ferramentas Photoscan da Agisoft, Remake e Recap 360 da Autodesk e Zephyr Free da 3Dflow foi possível verificar as semelhanças e diferenças de processamento e produtos gerados.

Com relação às semelhanças pode-se destacar que as quatro ferramentas testadas apresentam interfaces amigáveis, que facilitam seu uso e apreensão, e geraram produtos de elevada qualidade.

Com relação às diferenças, ressaltam-se as seguintes considerações. O modelo gerado pelo Photoscan destacou-se pela qualidade do modelo geométrico, pela rapidez do processamento e pela grande quantidade de opções de formatos de exportação. As ferramentas da Autodesk apresentaram comportamento similar, tendo a vantagem de não sobrecarregar o computador do usuário (com o seu processamento online), e de gerar produtos de qualidade, com tamanhos de arquivos reduzidos. O 3DF Zephyr Free apresentou os produtos em nuvem de pontos e malha TIN mais densos, o que acabou refletindo em maior tempo de processamento, em maiores tamanhos dos arquivos (comparados às outras ferramentas), exigindo máquinas mais robustas.

Através dos experimentos foi possível verificar a qualidade, a rapidez e a automação da técnica de restituição fotogramétrica Dense Stereo Matching. A técnica DSM representa o estado da arte da fotogrametria digital, sendo a mais adequada para o levantamento de formas complexas ou irregulares, permitindo gerar resultados de alta precisão e qualidade, de modo automatizado, com baixo custo operacional, caracterizando-se como um grande avanço para a documentação arquitetônica digital.

\section{AGRADECIMENTOS}

Os autores agradecem à Fundação de Amparo à Pesquisa do Estado da Bahia (FAPESB) pela bolsa de iniciação científica concedida através do programa PIBICUFBA, e ao LCAD/FAUFBA pelo apoio e infraestrutura concedida para o desenvolvimento da pesquisa.

\section{REFERÊNCIAS}

Amorim, A. L. (2017). A documentação arquitetônica como uma atividade multi, inter e transdisciplinar. Ponto de Acesso, Salvador, 11(1), 61-84.

Groetelaars, N. J. (2004). Um Estudo da Fotogrametria Digital na documentação de formas arquitetônicas e urbanas. 2004. 257 f. Dissertação (Mestrado em Arquitetura e Urbanismo) Faculdade de Arquitetura, Universidade Federal da Bahia, Salvador.

Groetelaars, N. J. (2015). Criação de modelos BIM a partir de "nuvens de pontos": estudo de métodos e técnicas para documentação arquitetônica. 2015. 372 f. Tese (Doutorado em Arquitetura e Urbanismo) - Faculdade de Arquitetura, Universidade Federal da Bahia, Salvador.

Hullo, J. F.; Grussenmeyer, P.; Fares, S. (2009). Photogrammetry and Dense Stereo Matching approach applied to the documentation of the cultural heritage site of Kilwa (Saudi Arabia).Proceedings of 22nd CIPA Symposium, Kyoto, Japan. Retrieved from http://www.photomodeler.com/applications/documents/Gruss enmeyerDSMScanning.pdf

Nikolov, I.; Madsen, C. (2016). Benchmarking Close-range Structure from Motion 3D Reconstruction Software Under Varying Capturing Conditions. Springer. Retrieved from https://doi.org/10.1007/978-3-319-48496-9_2

Oliveira, M. M.(2008) A Documentação como Ferramenta de Preservação da Memória. Brasília, D.F: IPHAN / Programa Monumenta.

Remondino, F.; Spera, M. G.; Nocerino, E.; Menna, F.; Nex, F. (2014). State of the Art in High Density Image Matching. The Photogrammetric Record 29 (146). Retrieved from https://doi.org/10.1111/phor.12063 